\title{
On the Importance of Purification of Sodium Polystyrene Sulfonate
}

\author{
Akhil K. Sen, ${ }^{1,2}$ Sandip Roy, ${ }^{1}$ and Vinay A. Juvekar ${ }^{1}$ \\ ${ }^{1}$ Department of Chemical Engineering, Indian Institute of Technology-Bombay, Powai, Mumbai 400076, India \\ ${ }^{2}$ Department of Chemical and Polymer Engineering, Birla Institute of Technology-Mesra, Ranchi 835215, India
}

Correspondence should be addressed to Akhil K. Sen, akhilsen@bitmesra.ac.in

Received 22 November 2011; Accepted 28 December 2011

Academic Editors: P. Kingshott and M. Volny

Copyright ( 2012 Akhil K. Sen et al. This is an open access article distributed under the Creative Commons Attribution License, which permits unrestricted use, distribution, and reproduction in any medium, provided the original work is properly cited.

\begin{abstract}
Ion exchange is commonly employed for purification of sodium polystyrene sulfonate (NaPSS), a molecule widely used as a model polyelectrolyte. However, the present work demonstrates that the ion exchange process itself may introduce some extraneous species into NaPSS samples by two possible mechanisms: (i) chemical transformation of polystyrene sulfonic acid (HPSS), a relatively unstable intermediate formed during ion exchange and (ii) release of small amount of "condensed" acid from cationic resins during the elution of NaPSS molecules. Based on these observations, it is proposed that simple dialysis is adopted as a standard protocol for the purification of primary NaPSS sample.
\end{abstract}

\section{Introduction}

Sodium polystyrene sulfonate (NaPSS) has been extensively used as a model polyelectrolyte system. It also has a wide variety of applications such as flocculation, personal care products, and drug, [1]. One of the critical applications involves its use as a drug during acute kidney failure; the oral dose for adult person is as high as $30 \mathrm{~g} /$ day [2]. The measurement of the properties of NaPSS as well as their prediction is useful from application point of view.

However, several workers [3-5] have indicated that commercial samples of NaPSS could contain ionic impurities at levels that may not be insignificant. Different types of possible impurities have been suggested: surface-active agents [3] or inorganic salts such as $\mathrm{Na}_{2} \mathrm{SO}_{4}$ [5]. The presence of the above impurities has strong effect on the polyelectrolyte properties like viscosity $[4,6-11]$, osmotic coefficiency [12$15]$, surface tension [16-19], and so forth. So the removal of the impurities from the commercial sample is essential.

As Tondre and Zana[20] have pointed out, the removal of ionic impurities from the polyelectrolyte system is not possible by conventional solution and precipitation technique; for such system dialysis followed by ion exchange method is necessary. Indeed, several researchers [5, 21, 22] have employed ion exchange method for purification of
NaPSS. However, as reported by Reddy and Marinsky[14], polystyrene sulfonic acid-an intermediate formed during ion exchange-is unstable at room temperatures, and thus any associated "degradation" process may inadvertently introduce impurities in the original sample, if proper care is not taken. In essence for experimental investigations, it is necessary to ensure that there are no additional substances present in a polyelectrolyte system such as NaPSS; or that, if present, they are trace levels, such that they do not affect the properties of the molecule.

In the present paper, we report experimental studies aimed at the standardizing the method of purification of NaPSS samples. The set of results reported here pertains to commercial samples of NaPSS obtained from M/s Sigma Aldrich (M.Wt. 70,000). We demonstrate that one of the sources by which "foreign" molecules may be introduced is a possible uncontrolled degradation of the polystyrene sulfonic acid (HPSS), which is formed as an intermediate during ion-exchange-based purification. This has already been suggested by Reddy and Marinsky [14]; however, we have subjected the phenomenon to a more detailed study here. In addition our experiment suggests that the ion exchange process itself may introduce ionic species not present in the original sample. In the opinion of the present authors a one-step dialysis of the parent NaPSS solution may 
constitute an adequate and reliable method of purifying NaPSS molecule after its synthesis.

\section{Materials}

The main materials used were sodium polystyrene sulfonate (molecular weight: 70,000), cation exchange resin (Amberlite 200), and anion exchange resin (Amberlite IRA 400C). Semipermeable membrane (cutoff molecular weight: 12,000) was used for dialysis of all NaPSS solutions. All the above materials were purchased from Sigma Aldrich Co., USA. Millipore water is used for dialysis and solution purposes.

\section{Experimental}

3.1. Dialysis of Commercial NaPSS Sample. NaPSS solution of concentration $50 \mathrm{~g} / \mathrm{L}$ was used for dialysis. The NaPSS solution was taken in sealed semipermeable membrane (cellulose acetate) and placed in distilled water. The external medium, that is, the dialyzate, was replaced from time to time (each replacement of water is referred as cycle) with fresh distilled water. The conductivity and UV absorbance of the dialyzate was measured at the end of each cycle. This was repeated until both the conductivity and UV absorbance of the dialyzate were practically equal to that of distilled water. The NaPSS solution within the membrane was finally evaporated to dryness in oven at $110 \pm 5^{\circ} \mathrm{C}$ to its solid form (this sample is referred as D-NaPSS). The dialyzate solution was similarly evaporated and the resulting powder (referred to as DM) used for further property measurements. The original (undialyzed) sample is referred to as UD-NaPSS.

3.2. Ion Exchange Method. Ion-exchange-based purification constitutes a critical step for removal of possible ionic impurities from the original NaPSS sample. Here we followed the procedure due to Reddy and Marinsky [14], which is as follows. The solution of D-NaPSS is first eluted through the anionic resin (Amberlite IRA 400C in hydroxide form) column for 4-5 cycles. The final elutant is next passed through cationic resin (Amberlite 200 in acid form, with five times weight equivalent of polyions) column, also for 4-5 cycles. Finally the resulting acid form of NaPSS is neutralized quantitatively with $\mathrm{NaOH}$ solution. The final solution was evaporated to dryness in oven at $110 \pm 5^{\circ} \mathrm{C}$ to its solid form. Before passing polyelectrolyte solutions, all the resins were thoroughly washed until they were free from acid or alkali.

Three separate types of NaPSS samples were collected from the above ion exchange process. Sample I is collected as the elutant from the anionic exchange resin column (alkaline in nature). The other two samples are collected from the cationic exchange resin column as follows. Sample II is collected just after the cation exchange process (HPSS) is over followed by neutralization with $\mathrm{NaOH}$. Lastly, a portion of the unneutralised elutant (HPSS) from the cation exchange column is left standing at $30^{\circ} \mathrm{C}$ for 24 hours and then neutralized with $\mathrm{NaOH}$ to obtain sample III. In summary all the three samples correspond to NaPSS solutions collected at different points of the ion exchange process.

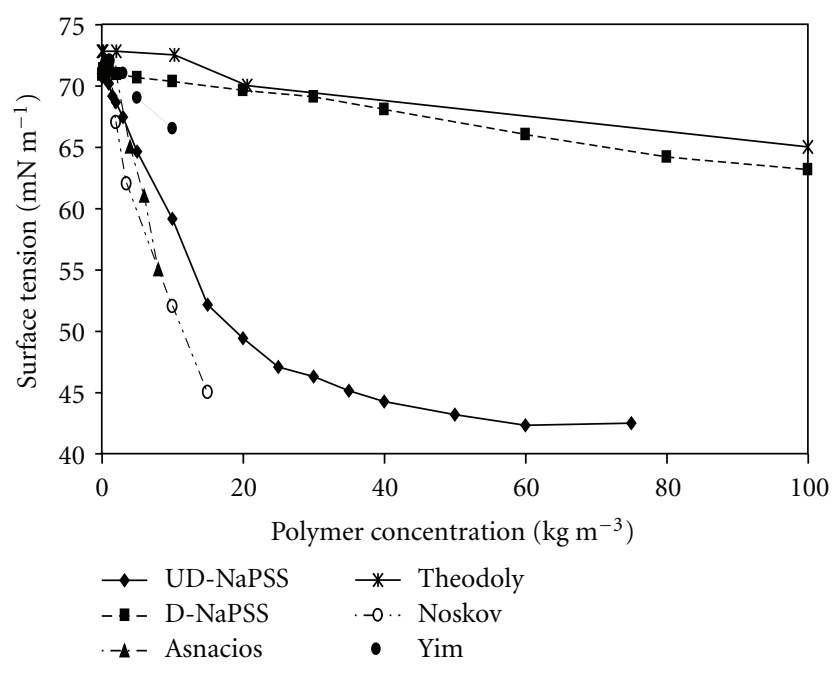

FIgURE 1: Surface tension of NaPSS solution.

3.3. Measurement of Osmotic Coefficient. Osmotic coefficient of the polyelectrolyte solutions was measured by Knorr vapor pressure osmometer (model K 7000) by dissolving solid NaPSS in distilled water. The instrument was first calibrated with a $\mathrm{NaCl}$ solution of known concentration and then the osmotic coefficient of polyelectrolyte solutions were measured. Further details are provided in [23].

3.4. Measurement of Surface Tension. The surface tension of different NaPSS solutions was measured using a Fischer Du Nouy ring tensiometer. All measurements were performed at $30^{\circ} \mathrm{C}\left( \pm 1^{\circ} \mathrm{C}\right)$. Since the attainment of equilibrium values of surface tension of NaPSS solution may take 1 to 3 hours depending on the solution concentration [24], prior to the actual surface tension measurement, each solution was equilibrated for at least three hours, following which the measurements were carried out at five-minute intervals (for about $30 \mathrm{~min}$ ). This ensured that the measured property corresponds to the equilibrium value.

\section{Results and Discussion}

4.1. Surface Tension of NaPSS Solution. The surface tension variation of UD-NaPSS and D-NaPSS sample of NaPSS are plotted in Figure 1. In the same plot similar data from the literature on NaPSS $[3,17,19]$ are also shown for comparison. Actual numerical data available with us show that none of the curves suggest surface activity of the polyelectrolyte concentrations below (less than) $1 \mathrm{~kg} / \mathrm{m}^{3}$. However as seen from Figure 1, at higher polymer concentrations, the DNaPSS sample from our work shows less surface activity than the corresponding UD-NaPSS sample. The surface tension exhibited by our D-NaPSS sample is almost similar to that from the work of Theodoly et al. [17]. However, results reported by Asnacios et al. [3], Yim et al. [19], and Noskov et al. [24] do not match with that of our D-NaPSS sample, rather they compare well with that of our UD-NaPSS sample. The reason for these disagreements is not obvious to the present authors. 


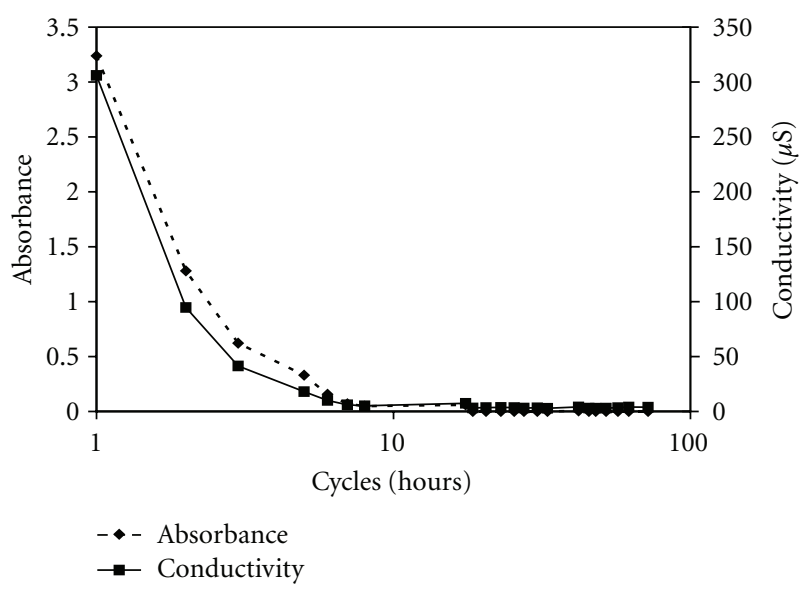

Figure 2: Absorbance and conductivity versus cycles (Hours) for dialyzate solution.

4.2. Analysis of DM Sample. The conductivity and absorbance of the DM solutions were checked for each cycle of water replacement. Figure 2 shows the values of conductivity and absorbance spectra of the DM solution after each cycle. As evident from the figure after $\sim 10-12$ dialysis cycles the conductivity and absorbance values (at $224 \mathrm{~nm}$ wave length) match those of distilled water and do not change thereafter. These results suggest that the DM is ionic in nature and is also UV active. Millipore water is used for dialysis purposes which has no absorption in the measured range $(200 \mathrm{~nm}$ to $300 \mathrm{~nm}$ ).

4.3. Osmotic Coefficient of NaPSS. Osmotic coefficient of a solution is dependent on the number of osmotically active particles in the solution. If a particular chemical (degradation) process increases the number of particles in the original solution, its osmotic coefficient will increase after degradation. The osmotic coefficients of different NaPSS (UDNaPSS, D-NaPSS, sample I, Sample II and sample III) samples from our work are plotted in Figure 3. The UD-NaPSS sample shows highest osmotic coefficient $(\sim 0.6)$, whereas the D-NaPSS sample shows the lowest $(\sim 0.2)$. The high osmotic coefficient of UD-NaPSS sample suggests possible presence of additional small (ionic) molecules as "impurity." The osmotic coefficient of NaPSS passed through anionic resin column (sample I) is almost similar to that of the D-NaPSS sample. This implies that no significant change in chemical composition of the NaPSS solution results from the passage through the anionic resin.

But the samples obtained from cationic resin column (sample II and sample III) have higher osmotic coefficients than that of the D-NaPSS sample. A higher osmotic coefficient suggests that the number of osmotically active particle is higher in both samples II and III. The enhancement in the osmotic coefficient in sample II implies that some changes in the solution composition may be taking place during the cationic exchange process itself. Also, the high osmotic coefficient of sample III suggests that HPSS formed in the cation exchange process is probably unstable at room temperature

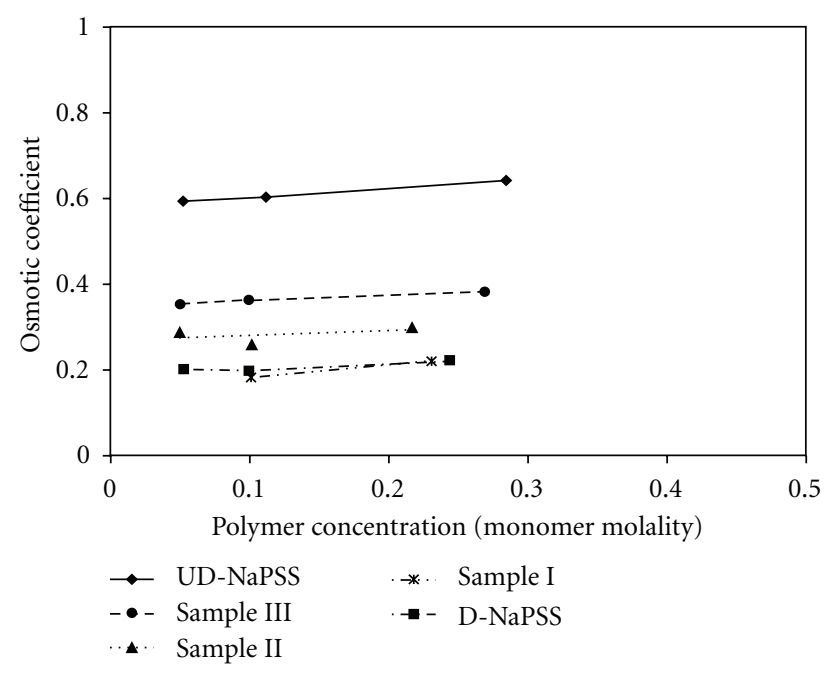

FIgURE 3: Osmotic coefficient of NaPSS solution.

and undergoes some form of transformation leading to creation of greater number of osmotically active particles. This is in agreement with the observation of Reddy and Marinsky [14], mentioned earlier in this paper. According to these authors such increase in osmotic coefficient may be due to various reactions that HPSS may undergo at room temperature such as degradation, crosslinking, sulfone formation.

Indeed, Reddy and Marinsky [14] have reported relatively high osmotic coefficients ( $\sim 0.6$ and above) of the degraded NaPSS sample, which are also similar to those reported by Ise and Okubo [13]. In their paper Reddy and Marinsky do not explicitly mention the conditions used for degradation. Nevertheless, they have suggested that the high osmotic coefficient of Ise and Okubo is also possibly due to degradation of HPSS solution during purification. We next investigated if cation exchange process could also directly introduce any ionic species in the NaPSS solution.

4.4. Osmotic Coefficient of Eluted NaPSS Samples. If indeed the ion exchange resin is the source of any extra ionic species then the variations in the inlet NaPSS solution concentration during elution may impact the elutant composition. To verify this we measured the osmotic coefficient of two eluted NaPSS samples that were obtained as follows. Two NaPSS solution of concentration $10 \mathrm{~kg} / \mathrm{m}^{3}$ and $20 \mathrm{~kg} / \mathrm{m}^{3}$, respectively, were eluted through cationic resin beds of the same loading. The elutant was neutralized quantitatively with $\mathrm{NaOH}$ and then evaporated to dryness in both instances. The solid obtained from the first case is referred to as sample $\mathrm{X}$, while that obtained in the latter case is termed sample $Y$. The osmotic coefficients of samples $\mathrm{X}$ and $\mathrm{Y}$ were in turn measured by preparing solutions of varying concentration. The results are plotted in Figure 4. In both the cases the osmotic coefficient is higher than that of D-NaPSS. In addition the osmotic coefficient of sample Y derived from original NaPSS of $20 \mathrm{~kg} / \mathrm{m}^{3}$ sample is higher compared to that of sample $\mathrm{X}$ obtained from NaPSS solution of $10 \mathrm{~kg} / \mathrm{m}^{3}$ strength. In both the cases the time required for the entire process of purification (elution 


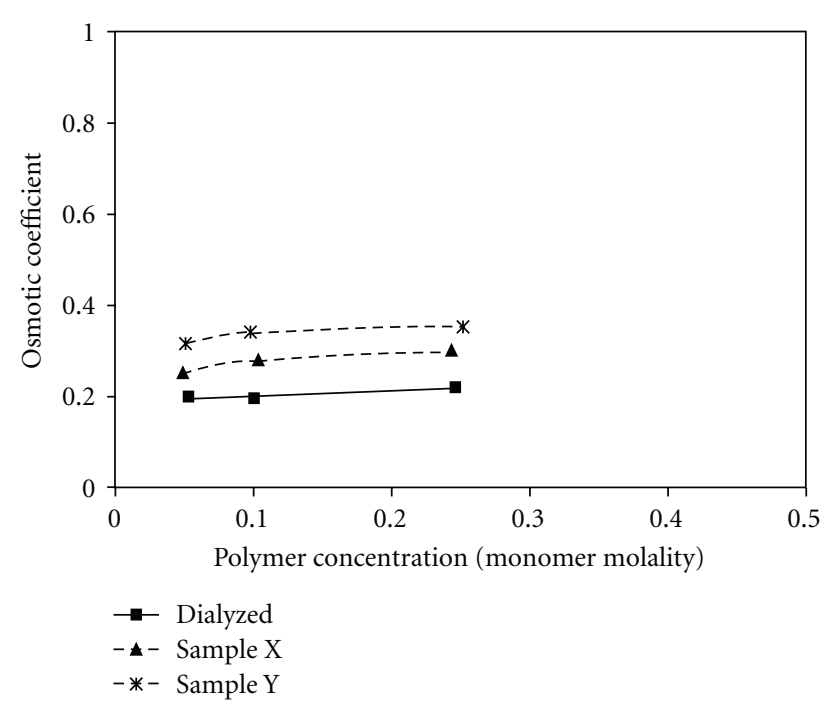

FIGURE 4: Osmotic coefficient of different eluted NaPSS sample.

and neutralization) was one and halfhour. These findings suggest that higher the presolution NaPSS concentration the greater is its capacity to release residual ions from the resin, which may be excess acid.

It is significant that these results are obtained after both the cationic and anionic resin columns were washed thoroughly with distilled water to remove any traces of free acid or alkali after the resin regeneration step. We have observed that the NaPSS solutions (sample I) become alkaline ( $\mathrm{pH}$ 10) after passing through the anionic resin column, but as evident from Figure 3, it has little or no effect on the osmotic coefficient. This suggests that the anionic resin-based purification may not be the likely source of any additional anionic substances in NaPSS samples.

One possible reason behind such a phenomenon may be as follow. The cation exchange resins are prepared by sulfonation of crosslinked polystyrene beads, which contains microchannels. After sulfonation this microchannels are expected to be highly charged, which can promote strong counterion $\left(\mathrm{H}^{+}\right)$condensation so that the high electrostatic repulsion between adjacent sites are reduced $[25,26]$. This excess (condensed) acid may be difficult to remove by simply washing with distilled water. Thus, when NaPSS solution is eluted through the acid form of cationic rein column, some acid molecules $(\mathrm{HCl})$ may be released from the microchannels and which may be replaced by NaPSS. The acid molecules so released can lead to the observed enhancement of osmotic coefficient of the NaPSS sample (i.e., sample II in this work).

In the present work we have used $\mathrm{HCl}$ for the regeneration of cationic resin. To check the possible presence of chloride ion in the eluted NaPSS sample we added silver nitrate solution to it. In case of sample II, the addition of silver nitrate solution gave white precipitate. However, following the regeneration step, the water eluting from the resin column (during the wash phase) was tested with litmus paper, which showed no presence of the acid. In contrast, the D-NaPSS solution does not show any white precipitate.
Thus, our data suggests that the higher osmotic coefficient of the final NaPSS solution may not only be due to possible degradation of the intermediate HPSS (at room temperature). It could also be due to the presence of extra acid molecules, which may be released from cation exchange resin column during the formation of HPSS intermediate.

\section{Conclusions}

The extraneous species in NaPSS may be introduced in two ways during ion exchange purification method: from direct chemical transformation of HPSS and from the cation exchange process. The former situation has also been suggested by other workers [14]. The introduction of additional ionic species by cation-resin-based purification process is possibly due to the following phenomenon. Some of the $\mathrm{HCl}$ used for regeneration of the cationic resin column may be present within the resin particles micropores even after thorough wash with distilled water. This is owing to strong counterion condensation required for decreasing electrostatic interactions between the adjacent ionic (sulfonated) sites on the pore walls. Since like the polyelectrolyte the cationic resin micropore wall sites are also sulfonated as NaPSS molecules elute through such a resin bed, during the ion exchange process part of the condensed acid may be released and part of HPSS retained.

It may thus be concluded that since resin purification method introduces some additional ionic species, simple dialysis is desirable for purification of NaPSS, which is capable of removing the majority of the extra chemical species present in the NaPSS solution. As shown in the present paper, 10-12 dialysis cycles involving replacement of the dialyzate by fresh distilled water alone can help purify NaPSS sample reliably.

The present work emphasizes that it is necessary to standardize the protocol for purification of NaPSS as its properties are known to be highly sensitive to the presence of other ionic species. This is necessary for ensuring accuracy and reliability of studies involving the polyelectrolyte, as it has several critical applications including that of healthcare.

\section{References}

[1] H. Dautzenberg, W. Jaeger, J. Kotz, B. Philipp, C. Seidel, and D. Stscherbina, Polyelectrolytes Formation, Characterization and Applications, Hanser Publishers, Munich, Germany, 1994.

[2] C. P. Walsh, F. R. Gittes, D. A. Perlmutter, and A. T. Stamey, Campbells Urology, vol. 3, W. B. Saunders, Philadelphia, Pa, USA, 5th edition, 1988.

[3] A. Asnacios, R. Klitzing, and D. Langevin, "Mixed monolayers of polyelectrolytes and surfactants at the air-water interface," Colloids and Surfaces A, vol. 167, no. 1-2, pp. 189-197, 2000.

[4] J. A. V. Butler, A. B. Robins, and K. V. Shooter, "The viscous behaviour of dilute solutions of a strong polyelectrolyte (Polystyrene Sulphonate)," Proceedings of the Royal Society of London A, vol. 214, no. 1226, pp. 299-310, 1957.

[5] M. Sedlák and E. J. Amis, "Dynamics of moderately concentrated salt-free polyelectrolyte solutions: molecular weight dependence," The Journal of Chemical Physics, vol. 96, no. 1, pp. 817-825, 1992. 
[6] D. C. Boris and R. H. Colby, "Rheology of sulfonated polystyrene solutions," Macromolecules, vol. 31 , no. 17, pp. 57465755, 1998.

[7] J. Cohen, Z. Priel, and Y. Rabin, "Viscosity of dilute polyelectrolyte solutions," The Journal of Chemical Physics, vol. 88, no. 11, pp. 7111-7116, 1988.

[8] F. R. Prini and E. A. Lagos, "Tracer diffusion, electrical conductivity, and viscosity of aqueous solutions of polystyrenesulfonates," Journal of Polymer Science A, vol. 2, no. 6, pp. 2917 2928, 1964.

[9] M. Rubinstein, R. H. Colby, and A. V. Dobrynin, "Dynamics of semidilute polyelectrolyte solutions," Physical Review Letters, vol. 73, no. 20, pp. 2776-2779, 1994.

[10] H. Vink, "Rheology of dilute polyelectrolyte solutions," Polymer, vol. 33, no. 17, pp. 3711-3716, 1992.

[11] J. L. M. S. Ganter, M. Milas, and M. Rinaudo, "On the viscosity of sodium poly(styrene sulphonate), a flexible polyelectrolyte," Polymer, vol. 33, no. 1, pp. 113-116, 1992.

[12] N. Ise and T. Okubo, "Mean activity coefficient of polyelectrolytes. V. Measurements of polyvinyl sulfates of various gegenions," The Journal of Physical Chemistry, vol. 71, no. 6, pp. 1886-1890, 1967.

[13] N. Ise and T. Okubo, "Mean activity coefficient of polyelectrolytes. VIII. Osmotic and activity coefficients of polystyrenesulfonates of various gegenions," Journal of Physical Chemistry, vol. 72, no. 4, pp. 1361-1366, 1968.

[14] M. Reddy and J. A. Marinsky, "A further investigation of the osmotic properties of hydrogen and sodium polystyrenesulfonates," The Journal of Physical Chemistry, vol. 74, no. 22, pp. 3884-3891, 1970.

[15] P. Chu and J. A. Marinsky, "The osmotic properties of polystyrenesulfonates. I. The osmotic coefficients," Journal of Physical Chemistry, vol. 71, no. 13, pp. 4352-4359, 1967.

[16] T. Okubo, "Surface tension of synthetic polyelectrolyte solutions at the air-water interface," Journal of Colloid And Interface Science, vol. 125, no. 2, pp. 386-398, 1988.

[17] O. Théodoly, R. Ober, and C. E. Williams, "Adsorption of hydrophobic polyelectrolytes at the air/water interface: conformational effect and history dependence," European Physical Journal E, vol. 5, no. 1, pp. 51-58, 2001.

[18] H. Yim, M. Kent, A. Matheson et al., "Adsorption of poly(styrenesulfonate) to the air surface of water by neutron reflectivity," Macromolecules, vol. 33, no. 16, pp. 6126-6133, 2000.

[19] H. Yim, M. S. Kent, A. Matheson et al., "Adsorption of sodium poly(styrenesulfonate) to the air surface of water by neutron and $\mathrm{x}$-ray reflectivity and surface tension measurements: polymer concentration dependence," Macromolecules, vol. 35, no. 26, pp. 9737-9747, 2002.

[20] C. Tondre and R. Zana, "Apparent molal volumes of polyelectrolytes in aqueous solutions," Journal of Physical Chemistry, vol. 76, no. 23, pp. 3451-3459, 1972.

[21] E. Hirose, Y. Iwamoto, and T. Norisuye, "Chain stiffness and excluded-volume effects in sodium poly(styrenesulfonate) solutions at high ionic strength," Macromolecules, vol. 32, no. 25, pp. 8629-8634, 1999.

[22] V. M. Prabhu, M. Muthukumar, G. D. Wignall, and Y. B. Melnichenko, "Polyelectrolyte chain dimensions and concentration fluctuations near phase boundaries," Journal of Chemical Physics, vol. 119, no. 7, pp. 4085-4098, 2003.

[23] A. K. Sen, Behavior of sodium polystyrene sulfonate in solution and at interfaces, Ph.D. thesis, Indian Institute of Technology, Bombay, India, 2005.
[24] B. A. Noskov, S. N. Nuzhnov, G. Loglio, and R. Miller, "Dynamic surface properties of sodium poly(styrenesulfonate) solutions," Macromolecules, vol. 37, no. 7, pp. 2519-2526, 2004.

[25] G. S. Manning, "Limiting laws and counterion condensation in polyelectrolyte solutions I. Colligative properties," The Journal of Chemical Physics, vol. 51, no. 3, pp. 924-933, 1969.

[26] A. K. Sen, S. Roy, and V. A. Juvekar, "Effect of structure on solution and interfacial properties of sodium polystyrene sulfonate (NaPSS)," Polymer International, vol. 56, no. 2, pp. 167$174,2007$. 


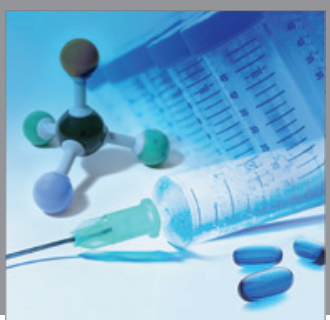

International Journal of

Medicinal Chemistry

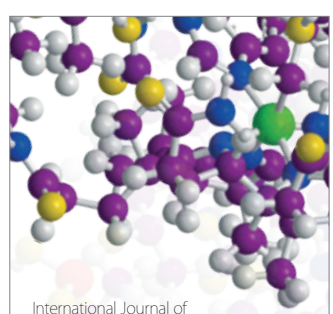

Carbohydrate Chemistry

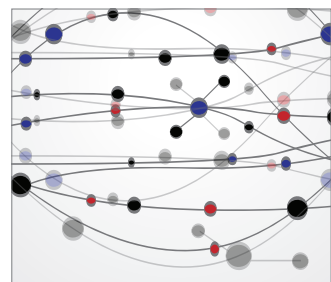

The Scientific World Journal
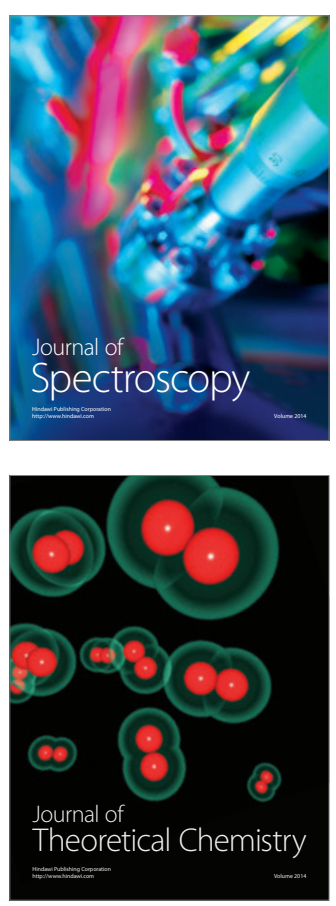
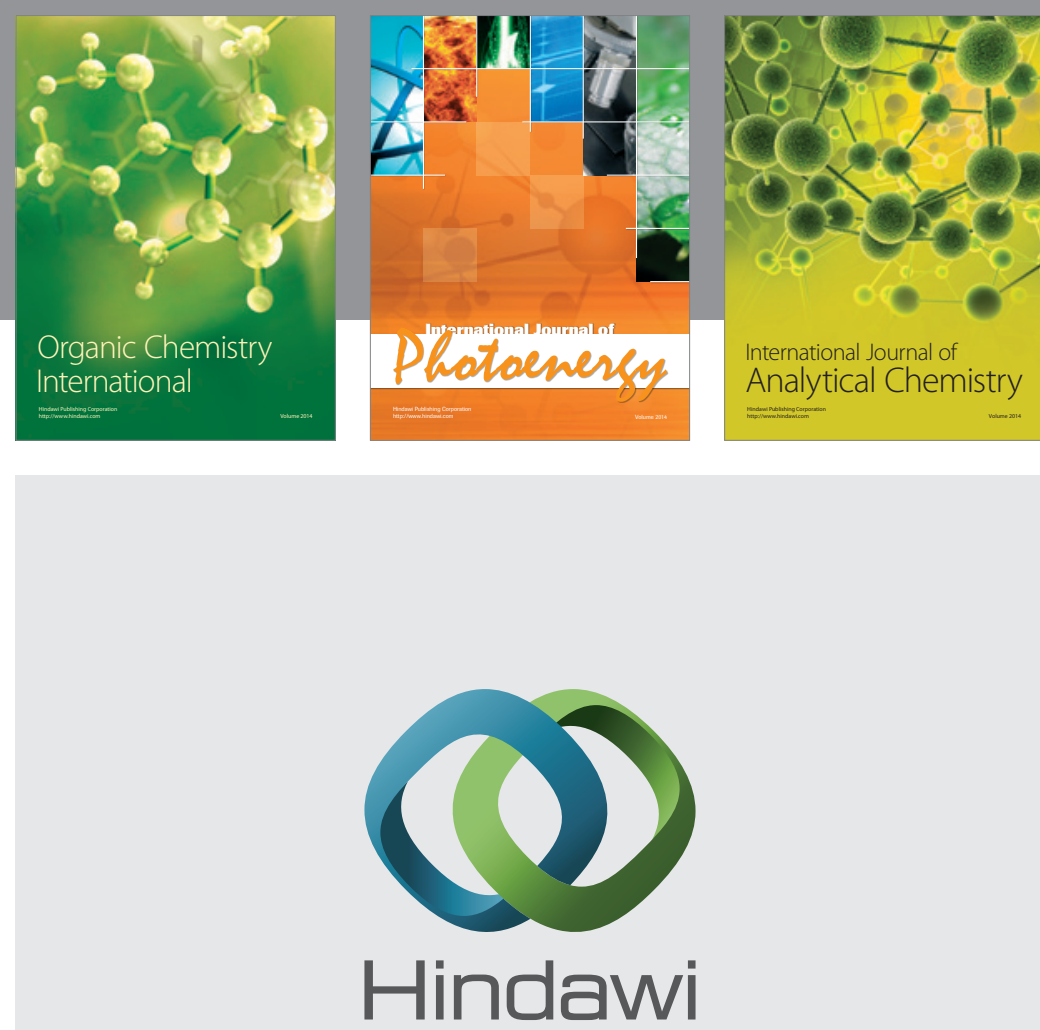

Submit your manuscripts at

http://www.hindawi.com
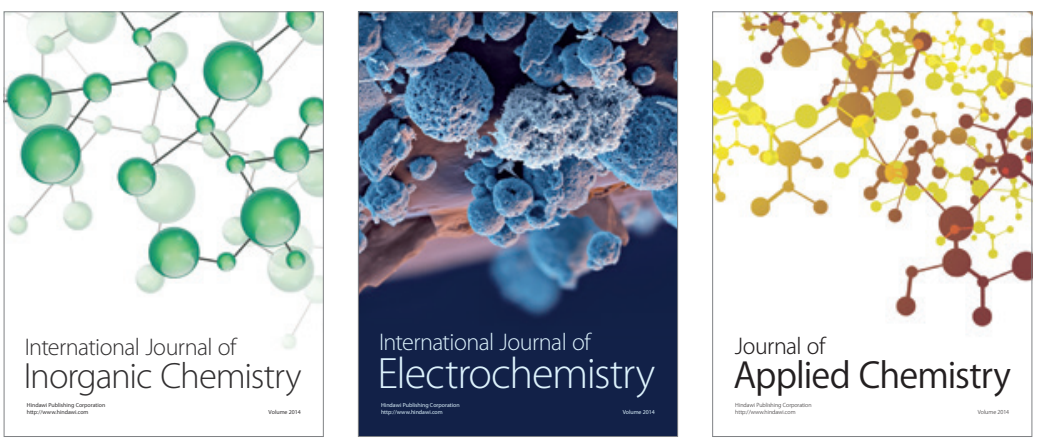

Journal of

Applied Chemistry
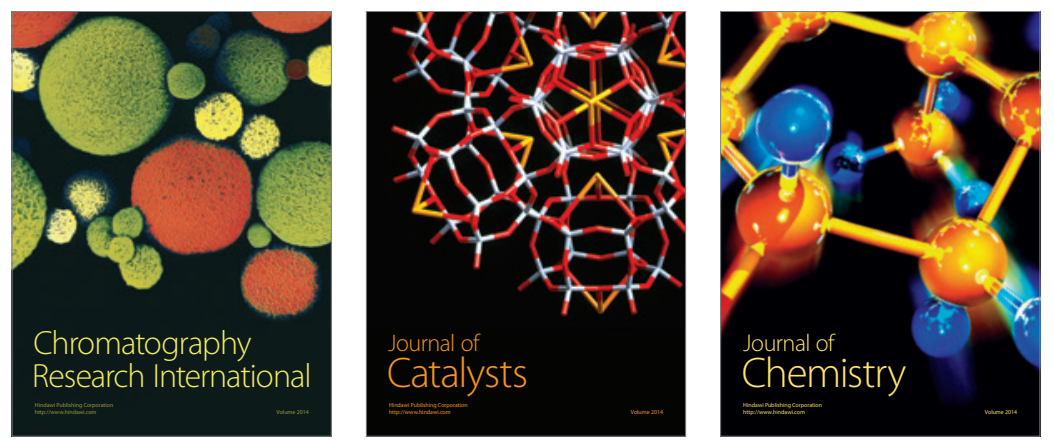
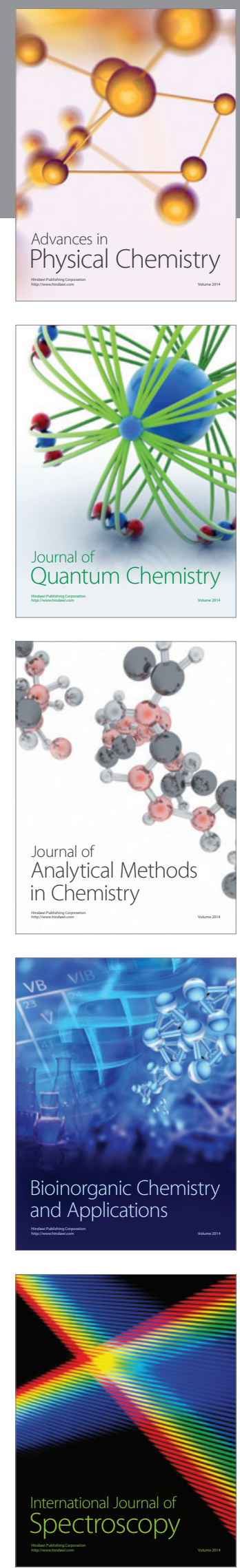